\title{
Energy and Angular Distributions of Electrons Emitted by Direct Double Auger Decay
}

\author{
Jens Vief haus, ${ }^{1, *}$ Slobodan Cvejanović, ${ }^{1}$ Burkhard Langer, ${ }^{2}$ Toralf Lischke, ${ }^{1}$ Georg Prümper, ${ }^{1}$ Daniel Rolles, ${ }^{1}$ \\ Alexander V. Golovin, ${ }^{3}$ Alexei N. Grum-Grzhimailo, ${ }^{1,4}$ Nikolai M. Kabachnik, ${ }^{1,4}$ and Uwe Becker ${ }^{1}$ \\ ${ }^{1}$ Fritz-Haber-Institut der Max-Planck-Gesellschaft ${ }^{\dagger}, 14195$ Berlin, Germany \\ ${ }^{2}$ Max-Born-Institut für Nichtlineare Optik und Kurzzeitspektroskopie, 12489 Berlin, Germany \\ ${ }^{3}$ Institute of Physics, St. Petersburg State University, 198504 St. Petersburg, Russia \\ ${ }^{4}$ Institute of Nuclear Physics, Moscow State University, 119992 Moscow, Russia
}

(Received 29 September 2003; published 26 February 2004)

\begin{abstract}
We have observed the direct $L_{2,3} M M M$ double Auger transition after photoionization of the $2 p$ shell of argon by angle-resolved electron-electron coincidence spectroscopy. The process is responsible for about $20 \%$ of the observed Auger electron intensity. In contrast to the normal Auger lines, the spectra in double Auger decay show a continuous intensity distribution. The energy and angular distributions of the emitted electrons allow one to obtain information on the electron correlations giving rise to the double Auger process as well as the symmetry of the associated two-electron continuum state.
\end{abstract}

DOI: 10.1103/PhysRevLett.92.083001

PACS numbers: $32.80 . \mathrm{Hd}, 32.80 . \mathrm{Dz}, 32.80 . \mathrm{Fb}$

An Auger process is the nonradiative rearrangement of the electronic structure of matter following an innershell vacancy production. Usually it leads to filling the vacancy in association with the emission of one of the outer electrons. The Auger process is studied in a wide range of basic and applied research fields from atomic physics to materials science [1]. Applied research uses Auger electron spectroscopy as a tool, because the emitted electrons have characteristic energies which are element specific. The latter is due to the fact that both the initial state (the innershell vacancy) as well as the final state with the vacancies in the outer shells have discrete energies. However, this is true only if one single electron was emitted in each Auger decay step. If two Auger electrons were emitted simultaneously, only the total kinetic energy is fixed, but the emitted electrons may share this energy arbitrarily. This direct double Auger decay involves electron correlations to a higher degree than the normal Auger process, since in the independent electron model (with frozen orbitals) emission of two electrons is forbidden. Therefore it is usually assumed to be a weak process. Because of the fact that it has to be described by a correlated two-electron wave function in the continuum, its study is both challenging as well as interesting from a fundamental point of view, particularly regarding the comparison with double photoionization (DPI) which is much better understood in its various details [2].

Measurements of double Auger decay started in the 1960s. The first evidence for double Auger electron ejection was found in $\mathrm{Ne}$ ion yield measurements, showing a sizable amount of triply charged final states [3]. Studies of the Auger electron spectra in Ar followed [4], where an Auger electron continuum was measured which was suggested to result from the double Auger process. In order to overcome the difficulty of discriminating this continuum from other sources, particularly from background electrons, coincidence studies are required. Ion-electron co- incidence measurements initiated by Krause et al. [5] were performed in several laboratories [6-8] as a necessary step in order to corroborate the findings of Carlson and Krause and to further investigate the role of the double Auger decay in Auger cascades [9]. Only measurements detecting the two electrons in coincidence could identify whether the direct double Auger process is indeed the dominant decay mechanism leading to triply ionized final states. The measurement of Hindi et al. [10] utilized the electron capture process to create a $K$-shell vacancy in argon. Using retarding field analyzers, they were able to confirm the double Auger process, but could make-due to the limited energy resolution - only rough estimates on the branching ratios and the corresponding energy distribution.

In this Letter, we present first angle-resolved electronelectron coincidence measurements of the direct double Auger process in Ar induced by photoionization. Our measurements were performed at BESSY (beam line UE56/2 PGM-1) and HASYLAB (beam line BW3SX700). The experimental setup is sketched in Fig. 1.

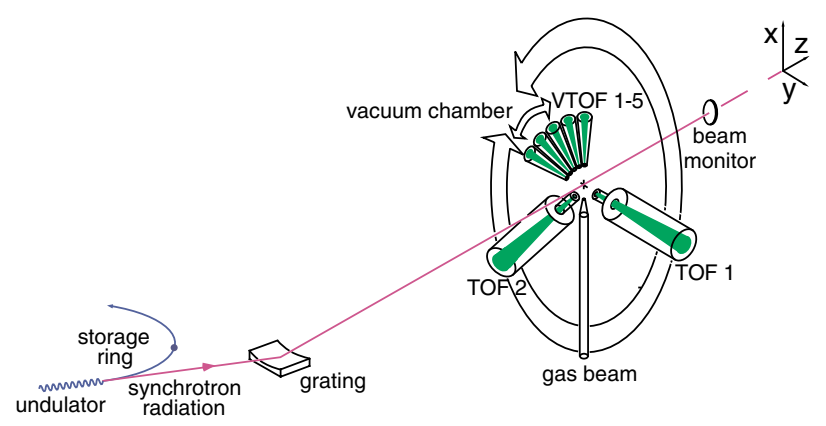

FIG. 1 (color online). A sketch of the experimental setup used for multiple time-of-flight electron-electron coincidence spectroscopy. 
Monochromatic synchrotron radiation crosses an effusive beam of Ar gas (99.999\% purity). Electrons produced in the interaction region are analyzed using the angleresolved time-of-flight technique [11]. A combination of six active analyzers mounted in the plane perpendicular to the incoming photon beam acquires both noncoincidence as well as coincidence spectra for all combinations of the detector pairs simultaneously. Two types of analyzers with different drift-tube lengths $(\approx 450$ and $\approx 150 \mathrm{~mm}$ ) were used, resulting in the relative energy resolution of $\approx 1 \%-2 \%$ and $\approx 3 \%-6 \%$, respectively.

The transmission of the analyzers was determined using Ne calibration spectra covering the energy region of interest and normalizing the $\mathrm{Ne} 2 p$ and $2 s$ lines with the known cross sections and angular distributions [12]. Very similar results were obtained using a set of electron spectra of the Ar 2p photoelectron line [13] and the corresponding Ar LMM Auger lines. The photon energy was calibrated using the literature values [14] for the Ar $2 p$ inner shell resonances. The photon bandpass was determined to be $\leq 200 \mathrm{meV}$, which allows one to resolve the fine structure of the Ar $2 p$ photoelectron lines. After the data collection, all spectra were converted from the time to the energy coordinate and corrected for transmission effects. In the case of the coincidence spectra, random coincidences were subtracted via an algorithm using the corresponding noncoincident spectra.

An overview of the main experimental results, obtained by summing up the coincidence spectra of all angle combinations, is given in the central panel of Fig. 2. The side panels show two representative noncoin-

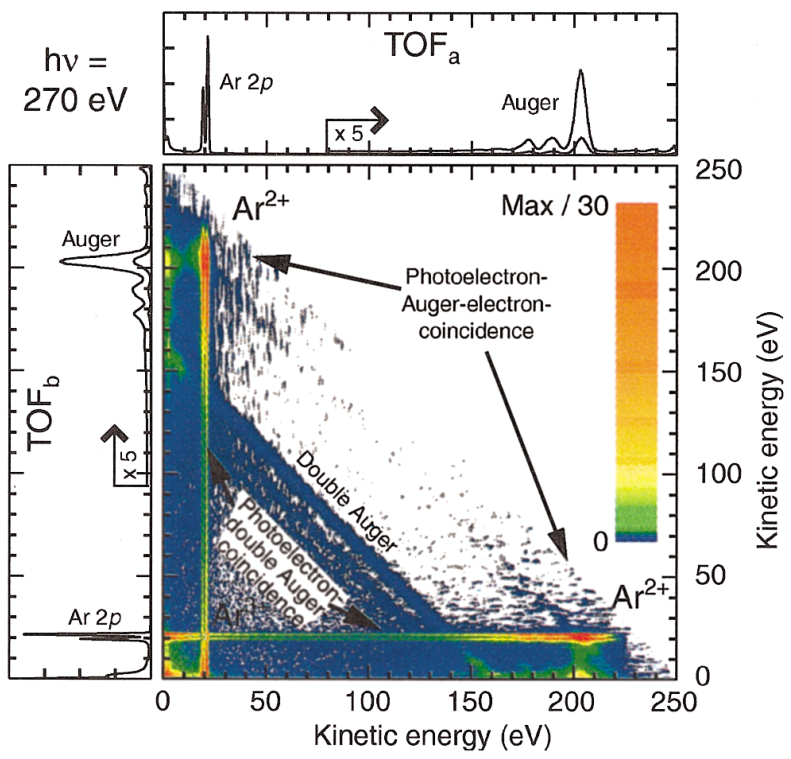

FIG. 2 (color). Two-dimensional electron-electron coincidence spectrum of Ar taken at $h \nu=270 \mathrm{eV}$ along with two noncoincident spectra. Structures due to normal Auger decay are marked by $\mathrm{Ar}^{2+}$. Diagonal stripes in which the sum of the kinetic energies is constant are caused by the double Auger electrons. cidence spectra. As expected, the normal Auger process is dominant and produces $\mathrm{Ar}^{2+}$ states seen as two rather strong structures located at the upper left and lower right corners of the coincidence map. Because of energy conservation, all triply charged states have to be located in a triangular region where the total kinetic energies are lower than those associated with the $\mathrm{Ar}^{2+}$ states. The double Auger continuum is observed as diagonal stripes in which the sum of the kinetic energies of the Auger electrons is constant. At least three different diagonal stripes are visible due to different electron excitations in the final state. Based on energy considerations, we attribute these stripes to the $3 p^{-3}$ and two groups of $3 s^{-1} 3 p^{-2}$ excitations. Our energy resolution is not sufficient to resolve different final state multiplets. Further features in the map are the horizontal and vertical lines extending to zero kinetic energy which are due to coincidences between the $\operatorname{Ar} 2 p$ photoelectron and one of the double Auger electrons.

For quantitative results on the branching ratio, we integrate the coincidence intensity along the diagonals, where the sum of the electron kinetic energies is constant. The corresponding representation (Fig. 3) shows the contribution of the doubly and triply charged final states. Taking into account the continuous intensity which is due to photoelectron-double-Auger coincidences, we obtain a branching ratio of $13(2) \%$ for the decay into triply charged states. This value is in full agreement with the ion results $[6,8]$ corroborating the interpretation of the coincidence spectrum from Fig. 2 as being equivalent to the angle integrated measurement despite the fact that our detectors cover only a fraction of the full solid angle.

The coincident registration of electrons allows us to select groups of $\mathrm{Ar}^{3+}$ final states and then analyze their

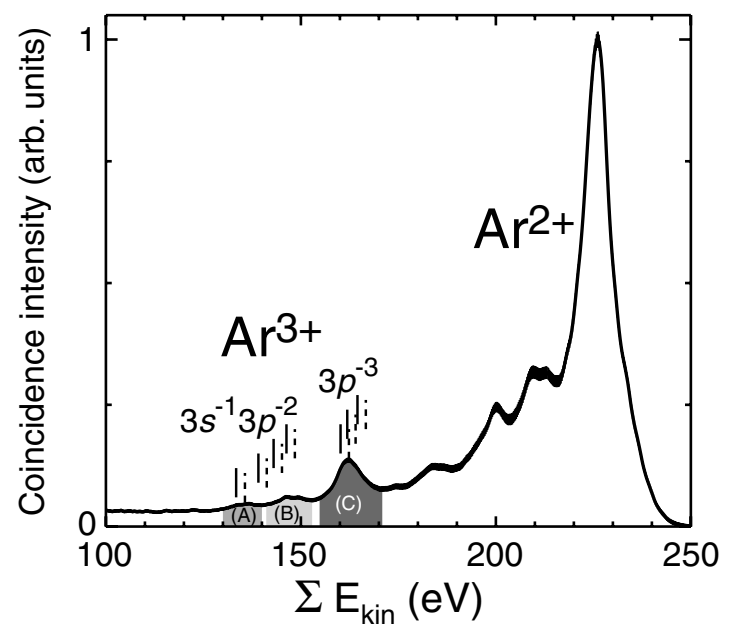

FIG. 3. Summed coincidence spectrum of Ar taken at $h \nu=$ $270 \mathrm{eV}$ showing different doubly and triply charged final states. The line thickness represents the statistical error. The energy positions of $\mathrm{Ar}^{3+}$ states obtained from [14,15] are indicated as bars (Ar $2 p_{3 / 2}$; full line: Ar $2 p_{1 / 2}$; dashed line). Line groups of further interest (see Fig. 4) are grey shaded. 
intensities with respect to the energy sharing of the two electrons. Figure 4 shows the result for three selected groups. In all cases, we observe a broad intensity continuum which is rising towards lower kinetic energies. This indicates that the two electrons favor an asymmetric energy sharing, in essential agreement with theoretical calculations [16]. Additionally, we observe discrete line structures in the low kinetic energy area which is superimposed on the direct double Auger continuum, especially for the $\operatorname{Ar} 3 p^{-3}$ states. This is a clear signature of cascade processes competing with the direct double Auger for the creation of the Ar $3 p^{-3}$ final states which could not be observed previously. For the two groups of Ar $3 s^{-1} 3 p^{-2}$ states, these structures are less prominent. In total, the cascade processes have a branching ratio of 26(4)\% for the $\mathrm{Ar}^{3+}$ final states.

Summarizing the spectroscopic findings of our work, we conclude that for impact of $270 \mathrm{eV}$ photons direct double Auger is the dominant decay mechanism for the creation of triply charged ions. With respect to the total Auger electron yield, it is responsible for about $20 \%$ of the intensity, due to the fact that two Auger electrons are emitted for each $\mathrm{Ar}^{3+}$ ion produced. Furthermore, we can clearly identify an additional contribution of cascade processes which in the first step undergo a transition to a highly excited intermediate $\mathrm{Ar}^{2+}$ state before decaying further in a second step. These intermediate states require a configuration with three holes and an excited electron. As the Rydberg states of this excited electron will progress towards a triply charged final state, only a transition to $\mathrm{Ar}^{3+}$ states of

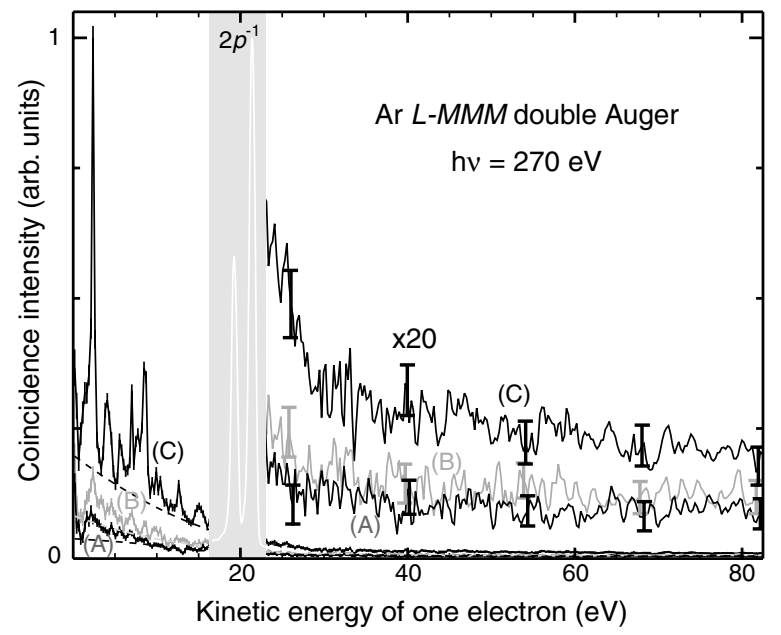

FIG. 4. Coincident energy distribution of the double Auger intensity for three different groups (shaded regions in Fig. 3) of triply charged final states $\left[3 s^{-1} 3 p^{-2}\right.$ : (A) total kinetic energy $\Sigma E_{\text {kin }}=135 \pm 5 \mathrm{eV}$, dark grey line; (B) $\Sigma E_{\text {kin }}=147 \pm 6 \mathrm{eV}$, light grey line. $3 p^{-3}$ : (C) $\Sigma E_{\text {kin }}=163 \pm 8 \mathrm{eV}$, black line]. For the scaled curves only representative error bars are shown. The dashed lines indicate the contribution of direct double Auger emission. The grey shaded area marks the region where the Ar $2 p$ lines interfere. lower binding energy is possible. This explains our observation of a progressively smaller cascade probability for the more tightly bound states.

With our experimental arrangement, we were able to study the angular correlations between the two emitted electrons in the double Auger process. The coincidence yield for each detector combination (i.e., various mutual angles $\theta_{a b}$ ) was first separated into the resolved final state groups, then binned into suitably chosen kinetic energy intervals to improve the statistical accuracy. For the case of $\operatorname{Ar} 3 p^{-3}$ states, the corresponding angular correlation patterns are displayed in Fig. 5 for four energy-sharing conditions, with the almost equal sharing case to the right, and progressively more unequal sharing cases to the left.

Since the symmetry structure of the electron wave function associated with a particular ionic state is more complex than for the He DPI case where only the ${ }^{1} P^{o}$ term of the two-electron continuum contributes, one could expect a big difference between the angular correlations in Ar double Auger and in He DPI, respectively $[17,18]$. To enable their qualitative comparison, the measured distributions and the simulations of the He triple differential cross sections (TDCS) are shown in Fig. 5 for the same kinematical conditions. The full line is obtained by using the parametrization model for the helium amplitudes [19] and an empirical interpolation of its parameters based on fitting all available He TDCS data at different kinematical conditions. The simulated curve is given for unpolarized light as the present measurements on Ar with circularly polarized light showed no significant dichroism effects.

According to our analysis [17], double electron continuum states contributing to the angular patterns of Fig. 5 fall into two broad classes: (i) those which have a node at $\theta_{a b}=180^{\circ}\left({ }^{1,3} P^{e}\right.$ and $\left.{ }^{1,3} F^{e}\right)$; (ii) those which have no node at that angle $\left({ }^{1,3} S^{e}\right.$ and $\left.{ }^{1,3} D^{e}\right)$ and whose angular correlation pattern is mainly determined by Coulomb repulsion of the two electrons. Such patterns are usually approximated by a Gaussian distribution (for the He DPI case, see [2]). The broken line in Fig. 5 shows a maximal contribution of the Gaussian-like distributions to the nodal angular pattern, showing that the ${ }^{1,3} P^{e}$ and ${ }^{1,3} F^{e}$ symmetries strongly dominate over the ${ }^{1,3} S^{e}$ and the ${ }^{1,3} D^{e}$. Another observed departure between our measurement and the He simulation concerns the most asymmetric energy-sharing case, where in the region of small mutual angles the Coulomb-repulsion assisted minimum in $\mathrm{He}$ is washed out. Besides the dynamical reasons for such a behavior, there may be a contamination of the measured yield by contributions which result in uncorrelated electrons. A closer inspection of the corresponding trace in Fig. 4 shows indeed a sudden rise of the yield below $30 \mathrm{eV}$, favoring the latter assumption. Because of the strong contribution of cascade processes, it was not possible to measure the angular correlations for the strongly asymmetric case $\left(E_{a} \ll E_{b}\right)$, where theory 


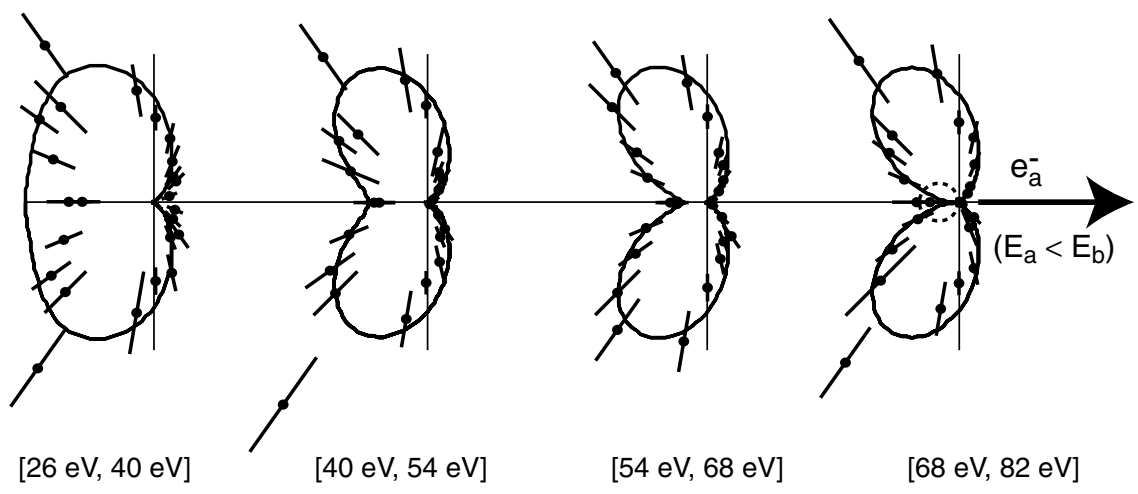

FIG. 5. Angular correlation patterns summed over all $\mathrm{Ar} 3 p^{-3}$ final ionic states (total kinetic energy $\Sigma E_{\text {kin }}=163 \pm 8 \mathrm{eV}$ ). The slower electron $e_{a}$ is emitted in the $x$ direction indicated by an arrow. The numbers in brackets give the corresponding kinetic energy range of the slower electron. The curves are explained in the text.

predicts an almost forward-backward symmetrical distribution [16].

This strikingly close similarity between the TDCS shapes in the Ar double Auger decay to the unresolved group of the $\left(3 p^{-3}\right)$ states and the He DPI is entirely unexpected, concerning both the pattern at near-equal energies and its evolution with energy sharing. Further theoretical investigation should elucidate whether this similarity is a pure coincidence or rather the manifestation of a more fundamental relationship.

In conclusion, we have used electron coincidence spectroscopy to unambiguously identify direct double Auger decay as the main rearrangement process after $\operatorname{Ar} 2 p$ innershell ionization leading to the formation of $\mathrm{Ar}^{3+}$ ions. The achieved experimental accuracy and resolution enabled us to gain a further insight into this three-body Coulomb process by studying the angular correlation patterns of the double Auger continuum. Despite the complexity of the symmetries of the two-electron wave functions involved, close similarities to the much simpler case of He double photoionization were observed.

The support of the BESSY and HASYLAB staff and, in particular, by Willy Mahler, Ralph Dörmann, and Thomas Möller, as well as the assistance of Christian Wienberg during the BESSY measurements, is gratefully acknowledged. The project is supported in part by the Bundesministerium für Bildung und Forschung $(05 \mathrm{KS} 1 \mathrm{~EB} 1 / 2)$ and the Deutsche Forschungsgemeinschaft (VI208/2-1). A. N. G. and N. M. K. are grateful to the Fritz-Haber-Institut der Max-Planck-Gesellschaft for hospitality and financial support.

*Electronic address: viefhaus@fhi-berlin.mpg.de ${ }^{\dagger}$ URL: http://www.fhi-berlin.mpg.de/

[1] W. Mehlhorn, J. Electron Spectrosc. Relat. Phenom. 93, 1 (1998).
[2] J. S. Briggs and V. Schmidt, J. Phys. B 33, R1 (2000).

[3] T. A. Carlson and M. O. Krause, Phys. Rev. Lett. 14, 390 (1965).

[4] T. A. Carlson and M. O. Krause, Phys. Rev. Lett. 17, 1079 (1966).

[5] M. O. Krause, M. L. Vestal, WH. Johnston, and T. A. Carlson, Phys. Rev. 133, A385 (1964).

[6] T. Hayaishi, E. Murakami, A. Yagishita, F. Koike, Y. Morioka, and J. E. Hansen, J. Phys. B 21, 3203 (1988).

[7] B. Kämmerling, B. Krässig, and V. Schmidt, J. Phys. B 25, 3621 (1992).

[8] S. Brünken, C. Gerth, B. Kanngießer, T. Luhmann, M. Richter, and P. Zimmermann, Phys. Rev. A 65, 042708 (2002).

[9] P. Lablanquie, S. Sheinerman, F. Penent, R. I. Hall, M. Ahmad, Y. Hikosaka, and K. Ito, Phys. Rev. Lett. 87, 053001 (2001).

[10] M. M. Hindi, L. Zhu, R. Avci, P. M. Miočinović, R L. Kozub, and G. J. Lapeyre, Phys. Rev. A 53, R3716 (1996).

[11] J. Viefhaus, G. Snell, R. Hentges, M. Wiedenhöft, F. Heiser, O. Geßner, and U. Becker, Phys. Rev. Lett. 80, 1618 (1998)

[12] F. Wuilleumier and M. O. Krause, J. Electron Spectrosc. Relat. Phenom. 15, 15 (1979).

[13] D. W. Lindle, L. J. Medhurst, TA. Ferrett, P. A. Heimann, M. N. Piancastelli, S. H. Liu, D. A. Shirley, T. A. Carlson, P. C. Deshmukh, G. Nasreen, and S.T. Manson, Phys. Rev. A 38, 2371 (1988).

[14] G. C. King, M. Tronc, FH. Read, and R C. Bradford, J. Phys. B 10, 2479 (1977).

[15] National Institute of Standards and Technology (NIST) Atomic Spectra Database Energy Levels Data available online at http://physics.nist.gov/cgi-bin/AtData/levels_ form (2003).

[16] M. Y. Amusia, I. S. Lee, and V. A. Kilin, Phys. Rev. A 45, 4576 (1992).

[17] A. N. Grum-Grzhimailo and N. M. Kabachnik, J. Phys. B (to be published).

[18] F. Maulbetsch and J. S. Briggs, J. Phys. B 28, 551 (1995).

[19] S. Cvejanović and T. J. Reddish, J. Phys. B 33, 4691 (2000). 